Cómo citar este artículo en Chicago: Mancilla Muñoz, Mauricio. "La actualidad del método hermenéutico de Friedrich Schleiermacher”. Escritos 29, no. 62 (2021): 56-72. doi: http://dx.doi.org/10.18566/escr.v29n62.a04

Fecha de recepción: 26.01 .2021

Fecha de aceptación: 01.03.2021

\title{
La actualidad del método hermenéutico de Friedrich Schleiermacher ${ }^{*}$
}

\author{
Topicality of the Hermeneutic Method of Friedrich Schleiermacher
}

\author{
Mauricio Mancilla Muñoz ${ }^{1}$
}

\section{RESUMEN}

Este artículo tiene como objetivo exponer la actualidad del método hermenéutico de Friedrich Schleiermacher (1768-1834) y mostrar su pertinencia para las investigaciones en el campo de las ciencias humanas. Uno de los supuestos advierte que la mayoría de los comentarios contemporáneos que se realizan a su programa hermenéutico parten de una "posición acrítica" y reproducen los supuestos trazados por la "lectura dominante" de Hans-Georg Gadamer. Esto fue posible por la falta de una "edición crítica” de la obra de Schleiermacher en relación con su hermenéutica, lo cual ha sido recientemente revertido con la publicación del volumen que aglutina sus Vorlesungen zur Hermeneutik und Kritik, que contiene un extenso número de notas y materiales adicionales. Se analizarán, a la luz de la edición crítica, algunos de sus conceptos fundamentales, como lenguaje, interpretación gramatical, interpretación técnico-psicológica, método adivinatorio, método comparativo, círculo del comprender, reconstrucción del discurso y comprensión como tarea infinita. En cuanto al modo concreto de proceder, se considera un análisis conceptual del conjunto de obras de Schleiermacher y una interpretación que, como se acaba de señalar, no se dejará determinar por los así llamados textos clásicos de la filosofía hermenéutica. Finalmente, el artículo concluye con algunas reflexiones sobre las novedosas posibilidades de la hermenéutica de Schleiermacher para el mundo contemporáneo.

Palabras clave: Friedrich Schleiermacher; Hermenéutica; Comprensión; Lenguaje; Interpretación gramatical; Interpretación técnico-psicológica; Método adivinatorio; Método comparativo; Círculo hermenéutico; Kunstlehre.

\section{ABSTRACT}

The aim of the article is to show that Friedrich Schleiermacher's Hermeneutic method is still topical and relevant for research in the field of humanities. One of the arguments is that most of recent commentaries on his hermeneutic program are based in a "non-critical position" and reproduce the assumptions of Hans-

* Este trabajo forma parte del proyecto del Fondo Nacional de Desarrollo Científico y Tecnológico (Fondecyt) n ${ }^{\circ}$ 1161762 "Ética dialéctica de la interpretación: La actualidad de la hermenéutica romántica de Friedrich Daniel Ernst Schleiermacher".

1 Doctor en Filosofía por la Universidad Complutense de Madrid, España. Profesor del Instituto de Filosofía, Universidad Austral de Chile. Correo electrónico: mauriciomancilla@uach.cl. 
Georg Gadamer's "dominant reading". This is the consequence of a lack of "critical editions" of the work of Schleiermacher regarding Hermeneutics, an issue that has recently been compensated for with the publication of the volume that collects his Vorlesungen zur Hermeneutik und Kritik, which includes numerous notes and additional material. Based on this critical edition, the article addresses some of his most important concepts, such as language, grammatical interpretation, technical or psychological interpretation, divinatory method, comparative method, hermeneutic circle of understanding, reconstruction of discourse, and interpretation as an infinite task. It undertakes a conceptual analysis of the aforementioned collection and an interpretation that is not undermined by classical works of Hermeneutics. Finally, it offers some remarks regarding the novel possibilities of Schleiermacher's philosophy for our time.

Keywords: Friedrich Schleiermacher; Hermeneutics; Understanding; Language; Grammatical Interpretation; Technical-Psychological Interpretation; Divinatory Method; Comparative Method; Hermeneutic Circle; Kunstlehre.

\section{Introducción}

$\mathrm{H}$ asta hace poco tiempo atrás, las ideas de Friedrich Schleiermacher sobre la hermenéutica no eran tan reconocidas como sus famosas obras teológicas Sobre la religión (1799) y La fe cristiana (1822), y el estado de su publicación fue una de las razones. Aparte de sus dos conferencias ${ }^{2}$ pronunciadas ante el pleno de la Academia Prusiana de las Ciencias, que forman un todo coherente, están disponibles principalmente las reflexiones de Schleiermacher sobre hermenéutica, en forma de notas, fragmentos y aforismos, que constituyen parte del corpus de las lecciones (Vorlesungen) que dictó en las universidades de Halle y Berlín. A pesar de varios intentos, Schleiermacher, quien falleció a los 66 años, no logró editar su proyecto de una "hermenéutica general"; incluso, las citadas conferencias también quedaron inéditas. Se han hecho numerosas especulaciones que tratan de explicar las razones de esta renuncia; sin embargo, es probable que Schleiermacher no haya publicado esta parte de su obra, y en esto sigo la argumentación de Jean Grondin, por convicción de "auténtico romántico". ${ }^{3}$ Probablemente nunca estuvo del todo satisfecho con sus proyectos o con la manera de formularlos, lo cual queda reflejado por el incesante vacilar de su terminología y de los puntos generales sobre los que giran sus planteamientos. Además, como releva su propia concepción, nunca se llega a comprender un discurso del todo, sino que se trata de una tarea infinita. Su pensamiento hermenéutico nos ha llegado a través de la edición póstuma realizada por su discípulo Friedrich Lücke, a cuatro años de su muerte, bajo el título Hermenéutica y crítica con especial referencia al Nuevo Testamento (1838). ${ }^{4}$ La naturaleza fragmentaria de su obra no

2 Schleiermacher dictó estas dos conferencias entre el 13 de agosto y el 22 de octubre de 1829, con el mismo título que luego fueron publicadas: "Sobre el concepto de hermenéutica en relación con las indicaciones de F. A. Wolf y al manual de Ast”; cf. Lourdes Flamarique, Introducción a Los discursos sobre hermenéutica, de Friedrich Schleiermacher (Pamplona: Universidad de Navarra, 1999), 31.

3 Jean Grondin, Introducción a la hermenéutica filosófica (Barcelona: Herder, 2002), 109.

4 Cf. Friedrich Schleiermacher, Hermeneutik und Kritik mit besonderer Beziehung auf das Neue Testament (Berlín: Reimer, 1838). Durante más de un siglo, la edición de Friedrich Lücke resultó ser el único texto "canónico" para acceder a sus planteamientos hermenéuticos. En los últimos sesenta años, se han editado dos versiones que imprimen algunas variantes a este "canon". Heinz Kimmerle, en 1959, organizó y publicó nuevamente una pequeña parte de los manuscritos y materiales, y agregó "Apuntes", "Discursos académicos" y un conjunto de "Notas", con los que resaltó su carácter de "borrador" (Entwurf); cf. Friedrich Schleiermacher, Hermeneutik (Heidelberg: Abhandlungen der Heidelberger Akademie der Wissenschaften, 1959). Y poco más tarde, Manfred Frank, en 1977, presentó una nueva edición ampliada de los 
significa que Schleiermacher no tuviera in mente un concepto sistemático de hermenéutica. Al contrario, como ha mostrado muy bien Wolfgang Virmond en la reciente edición crítica, incluso ya los primeros aforismos de 1805, que exponen un esbozo ordenado de su hermenéutica, tienen continuidad hasta sus últimas lecciones de 1832-1833. ${ }^{5}$ Una lectura comparativa de sus manuscritos muestra que su enfoque siguió siendo el mismo a lo largo de su vida.

Sin embargo, el carácter póstumo de su obra no es la única dificultad que deben enfrentar los lectores de Schleiermacher, sino que una segunda razón proviene de un hecho histórico-filosófico. La mayoría de los comentarios contemporáneos que se realizan a este programa hermenéutico parten de una "posición acrítica" y reproducen los supuestos trazados por la "lectura dominante" de Hans-Georg Gadamer. La influencia de la órbita filosófica de Gadamer restringe la interpretación de los planteamientos de Schleiermacher. En su esbozo de la historia de la hermenéutica moderna, al comienzo de la segunda parte de Verdad y método, aborda la contribución de Schleiermacher bajo el título "Lo cuestionable de la hermenéutica romántica". ${ }^{6}$ Aunque Gadamer elogia su planteamiento como arte de evitar malentendidos y su reivindicación de la universalidad de la hermenéutica, critica su método adivinatorio como el despliegue de una "metafísica estética de la individualidad" (ästhetische Metaphysik der Individualität). ${ }^{7}$

En este artículo, nos gustaría reflexionar de nuevo sobre el impacto de la hermenéutica de Schleiermacher en la teoría de la interpretación contemporánea, más allá del rígido "corsé" impuesto por el juicio de Gadamer, sin promover acríticamente su pensamiento. Si bien la hermenéutica se ha convertido en los últimos años en un concepto clave para la reflexión filosófica y metodológica, y un requisito indispensable para una segura y correcta interpretación, nos parece que este término tiene, en su uso actual, un aura difusa; por ello, se considera que, a partir del programa de Schleiermacher, es posible proponer una nueva "lógica hermenéutica" que renueve las posibilidades de la interpretación para las ciencias humanas. Esto se sustenta en la vigencia teórica de los diferentes ámbitos de su obra y su riqueza para enfrentar los desafíos de una sociedad global y compleja.

\section{La generalidad del problema hermenéutico}

Friedrich Daniel Ernst Schleiermacher (1768-1834), nacido en Breslau (Silesia), fue hijo de un clérigo calvinista, que se educó en las escuelas moravas y luteranas bajo el signo del pietismo. ${ }^{8}$ En 1787, ingresó

manuscritos publicados por Lücke, junto con una serie importante de anexos; cf. Friedrich Schleiermacher, Hermeneutik und Kritik (Fráncfort del Meno: Suhrkamp, 1977). Recién, con la publicación en curso de Die Kritische SchleiermacherGesamtausgabe (citado a partir de ahora como $K G A$ ), que reúne la "totalidad" de sus escritos y correspondencias, iniciada en la década de 1980, se viene a subsanar un problema histórico en torno a la apropiación de su obra; cf. Friedrich Schleiermacher, Vorlesungen zur Hermeneutik und Kritik, KGA II/6 (Berlín: Walter de Gruyter, 2012).

5 Cf. Wolfgang Virmond, Introducción a Vorlesungen zur Hermeneutik und Kritik, VII-LII.

6 Hans-Georg Gadamer, Hermeneutik I: Wahrheit und Methode. Grundzüge einer philosophischen Hermeneutik (Tubinga: Mohr-Siebeck, 2010), 177.

7 Gadamer, Hermeneutik I, 193.

8 Cf. Walter Jaeschke y Andreas Arndt, Die Klassische Deutsche Philosophie nach Kant: Systeme der reinen Vernunft und ihre Kritik 1785-1845 (Múnich: Beck, 2012), 254. 
en la Universidad de Halle, donde cursó estudios superiores de Teología, Filología Clásica y Filosofía. Allí tuvo acceso a las obras de Aristóteles, Kant y Spinoza, que influyeron decisivamente en la formación de su pensamiento. ${ }^{9}$ Una vez instalado en Berlín, a partir de 1796, comenzó a trabajar como pastor y predicador reformado en el hospital de la Charité. En estos años, frecuentó varios salones literarios, en especial, el prestigioso salón de Henriette Herz, donde entabló amistad con algunos de los más significados representantes del romanticismo alemán. En una carta a su hermana Charlotte del 22 de octubre de 1797, Schleiermacher confiesa que allí conoció a un joven Friedrich Schlegel, ${ }^{10}$ con quien entabló una estrecha amistad y compartió, durante algún tiempo, un modesto departamento en la Oranienburger Tor, donde formaron una pequeña comunidad filosófica. ${ }^{11}$ Ambos fueron parte del movimiento filosófico que más tarde sería conocido bajo el nombre de romanticismo temprano (Frühromantik) y que se articuló en torno de la revista Athenaeum (1798-1800). Su obra Sobre la religión (1799) muestra con claridad su adhesión a los ideales del movimiento romántico y presenta una renovación de la religión, fundamentada en la "intuición" (Anschauung) y en el "sentimiento" (Gefühl). ${ }^{12}$

En 1804, fue llamado a la luterana Universidad de Halle para impartir clases de exégesis del Nuevo Testamento, así como de ética filosófica y teológica. Allí comenzó a traducir las obras de Platón, labor en la que estuvo comprometido durante más de treinta años, empresa que desempeñó un papel preponderante en el desarrollo de su pensamiento filosófico. Al comienzo de su trabajo académico, mientras preparaba un seminario de exégesis bíblica, recurrió a la obra del filólogo y teólogo pietista Johann August Ernesti, quien, en 1761, había publicado su Institutio interpretis Novi Testamenti. Schleiermacher rápidamente se dio cuenta de que esta obra, al igual que toda la literatura relevante en ese momento (así lo expresa en una carta a su amigo y profesor de teología, Joachim Christian Gaß), era bastante inadecuada como teoría de la interpretación. ${ }^{13}$ Este hecho lo llevó a desarrollar su propia propuesta hermenéutica.

A lo largo de su carrera, entre 1805 y 1833, Schleiermacher dictó nueve lecciones sobre hermenéutica. El "primer borrador" (erster Entwurf) de 1805, si bien sigue la antigua tradición teológica, en especial vinculada a la interpretación del Nuevo Testamento, expresamente no pretende ser una hermenéutica sacra, sino solo seguir las pautas filosóficas y filológicas que contiene. En 1807, regresó a Berlín para participar en el proyecto de creación de una nueva universidad, donde se desempeñó hasta el final de sus días. Allí volvió a abordar el problema de la interpretación con una orientación consciente hacia una posición universal. A partir del curso de 1809-1810, Schleiermacher imparte lecciones con el título de hermenéutica general, en que el objetivo es "la comprensión en el más alto sentido" (das Verstehn Im höchsten Sinne). Ahora bien, la tarea de la hermenéutica no solo puede comenzar cuando la comprensión se "vuelve incierta" (unsicher wird), sino que está conectada desde el principio con el intento de "querer entender un discurso" (eine Rede verstehn $z$ u wollen). ${ }^{14}$

9 Cf. Kurt Nowak, Schleiermacher: Leben, Werk und Wirkung (Gotinga: Vandenhoeck \& Ruprecht, 2001 ), 17.

10 Cf. Friedrich Schleiermacher, Briefwechsel 1796-1798. KGA V/2 (Berlín: Walter de Gruyter, 1988), 177.

11 Andreas Arndt, "Eine literarische Ehe: Schleiermachers Wohngemeinschaft mit Friedrich Schlegel”, en Schleiermacher als Philosoph (Berlín: Walter de Gruyter, 2013), 31.

12 Cf. Kurt Nowak, Schleiermacher und die Frühromantik: Eine literaturgeschichtliche Studie zum romantischen Religionsverständnis und Menschenbild am Ende des 18. Jahrhunderts in Deutschland (Gotinga: Vandenhoeck \& Ruprecht, 1986), 140 y ss.

13 Cf. Friedrich Schleiermacher, Briefwechsel 1804-1806, KGA V/8 (Berlín: Walter de Gruyter, 1988$), 67$.

14 Schleiermacher, Vorlesungen zur Hermeneutik und Kritik, 74. 
Schleiermacher señala que la necesidad de una hermenéutica es más urgente cuando la comprensión se ve obstaculizada. En este sentido, el uso espontáneo, no reflexivo, es decir, cotidiano de la comprensión, conduce inevitablemente a graves errores que debemos revisar y corregir mediante la generación de un "arte" (téchne). Si bien la necesidad de una hermenéutica se hace evidente ante la dificultad o imposibilidad de comprender un discurso, la tesis de Schleiermacher advierte que la interpretación desde el principio debe seguir reglas o pautas estrictas. Cualquier tipo de discurso tiene un lado naturalmente "incomprensible" (unverständlich) que nunca puede eliminarse por completo. Al hacer hincapié en que el "malentendido" (mißverstehen) "se produce desde sí mismo", el pensador berlinés apunta a la importancia esencial de diseñar y aplicar conscientemente un sistema de reglas, que, al mismo tiempo, destella la universalidad del fenómeno positivo del comprender y que "en cada punto debe ser querido y buscado". ${ }^{15}$ En síntesis, "la hermenéutica descansa en el factum de la no comprensión del discurso" (Nichtverstehns der Rede). ${ }^{16}$ Este malentendido, que se da en la lengua materna y en la vida común, se debe a la indeterminación o ambigüedad del contenido de todo discurso.

\section{El lugar del lenguaje en la comprensión}

Ya en las primeras notas del citado "borrador" de 1805 surgió una idea que Gadamer convirtió en lema para la tercera parte de Verdad y método: "Todo lo que hay que presuponer en la hermenéutica es únicamente lenguaje." ${ }^{17}$ Schleiermacher, unas líneas más abajo del mismo fragmento, profundiza en esta idea al señalar que en el lenguaje deberán ser encontrados los "presupuestos objetivos y subjetivos" (objektiven und subjektiven Voraussetzungen) ${ }^{18}$ que orienten la interpretación. La tarea hermenéutica se centra en la interpretación de un discurso, ya sea oral, ya sea escrito, producido por un individuo en el que se expresan sus pensamientos, sentimientos y deseos. En esto último, se pone en juego una concepción lingüística muy específica que debemos tratar de clarificar.

El lenguaje, así como sus manifestaciones sociales e históricas, es tratado por Schleiermacher como una expresión supraindividual en la medida en que lo singular-personal está enraizada en él. ${ }^{19}$ Gracias a esta concepción se hace posible la comunicación interpersonal, pero no se le debe reducir a una mera función trascendental. El lenguaje siempre es modificado por la acción creativa e ingeniosa de las personas y, por tanto, no se puede entender como una estructura estática. Cada persona es, de hecho, "un lugar en donde se configura un lenguaje ${ }^{20} \mathrm{y}$ donde un determinado sistema lingüístico se manifiesta de manera

15 Schleiermacher, Vorlesungen zur Hermeneutik und Kritik, 127.

16 Schleiermacher, Vorlesungen zur Hermeneutik und Kritik, 73.

17 Gadamer, Hermeneutik I, 387.

18 Schleiermacher, Vorlesungen zur Hermeneutik und Kritik, 17.

19 Como ha mostrado Denis Thouard, hay un gran trasfondo en la filosofía de Schleiermacher que entrelaza estrechamente pensamiento y lenguaje, y que está en relación con la moderna comprensión del lenguaje de Herder, Kant, Schelling y Wilhelm von Humboldt; cf. Denis Thouard, “Die Sprachphilosophie der Hermeneutik”, en Friedrich Schleiermachers: Hermeneutik, Interpretationen und Perspektiven, ed. por Andreas Arndt y Jörg Dierken (Berlín, Walter de Gruyter, 2016), 85-100.

20 Schleiermacher, Vorlesungen zur Hermeneutik und Kritik, 121. 
específica. Las palabras, por ejemplo, solo pueden ser entendidas en estrecha relación con el sistema lingüístico en su conjunto. El proceso de influencia que conecta al hombre con su lenguaje es de carácter circular, el cual está incrustado orgánicamente en la forma de vida de la comunidad y es transformado sucesivamente por los hablantes.

La relación entre el habla y la comprensión, en el pensamiento de Schleiermacher, es tan estrecha que "todo acto de comprender es la inversión del acto de hablar", lo cual, en el fondo, nos invita a tratar de llegar al pensamiento que "subyace a lo que se dice". ${ }^{21}$ Las personas conectan sus vidas, intereses y deseos, principalmente, a través de un lenguaje comúnmente hablado. Una manifestación lingüística no puede ser tratada en absoluto como una expresión de una persona si no la hemos considerado previamente a la vez como lingüística. El lenguaje en tanto acción comunicativa de los hablantes es tanto manifestación lingüística como expresión de una persona específica.

Para Schleiermacher, el lenguaje vivo, infinitamente diferenciado en expresiones individuales, ocupa un lugar central en su reflexión. Por esta razón, todo testimonio lingüístico, aunque en diferente grado, requiere un esfuerzo activo de comprensión. Schleiermacher exige que el proceso de formulación lingüística sea invertido a través de la interpretación ${ }^{22}$ hasta llegar a su origen. En el fondo, se trata de captarlo desde su interioridad. Sin embargo, el verdadero propósito de la actividad interpretativa no termina ahí. Se busca lo subjetivo-individual que puede presentar la singularidad y el genio del autor. Más allá de cualquier tipo de comprensión lingüística externa, según Schleiermacher, solo podemos entender el significado del texto si logramos revivirlo, experimentar los procesos mentales de su autor. El progreso sistemático de la comprensión emerge, en algunos aspectos, como una repetición retrospectiva e inversa del proceso creativo: a partir del resultado final, un texto o una obra de arte, tenemos que encontrar el camino de regreso a la vida mental creativa del autor (mens auctoris).

Para ello, ya no es suficiente, como había hecho la mayor parte de la literatura anterior, limitar el esfuerzo hermenéutico al análisis lingüístico de un texto. Comprender no es solo "comprender en el lenguaje", sino también "comprender en el habla", porque todo hablante es, por un lado, un órgano del lenguaje, en la medida en que este guía sus combinaciones intelectuales, y por otro, el lenguaje es también un órgano del hablante en tanto este enriquece el universo lingüístico a través de su experiencia y forma de pensar. ${ }^{23}$ Esa es una de las ideas más importantes de Schleiermacher en esta área. Por ello, divide sus lecciones en atención, por una parte, al aspecto gramatical, que se ocupa de la comprensión del lenguaje, y por otra, al aspecto técnico-psicológico, que se centra en la comprensión del autor. En la interpretación gramatical, lo humano se entiende desde el lenguaje, y en la interpretación técnico-psicológica, el lenguaje desde lo humano. Ambos deben estar relacionados y vinculados entre sí. Esta conexión hace que la hermenéutica sea un arte en contraste con un mero seguimiento mecánico de reglas. A continuación, examinaremos brevemente los puntos principales que sustenta este modo de proceder.

21 Schleiermacher, Hermeneutik und Kritik, 76.

22 Schleiermacher, Vorlesungen zur Hermeneutik und Kritik, 38.

23 Schleiermacher, Vorlesungen zur Hermeneutik und Kritik, 50. 


\section{Interpretación gramatical e interpretación técnico-psicológica}

Independiente de la dimensión filosófica que recibe la hermenéutica de Schleiermacher, a través de su justificación en el acto de comprender, nunca pierde de vista los problemas metodológicos y prácticos de la interpretación. Su conexión en torno al lenguaje recién esbozada implica inmediatamente una dicotomía en el ejercicio de la interpretación. Cada discurso puede verse desde una doble perspectiva: ya sea como un sistema que expresa las condiciones y limitaciones a las que está sometido el hablante, ya sea como un instrumento que se utiliza para expresar los pensamientos más personales. Según esto, la pregunta básica dual de la hermenéutica en Schleiermacher es la siguiente: ¿cómo hacemos para definir el significado del texto en cuanto tal? y ¿ cómo definimos la intención subjetiva del autor? Para responder a estas, habrá que hacerse cargo de las tareas relacionadas con su propuesta hermenéutica. Primero, hay que entender la interpretación de las manifestaciones lingüísticas como expresiones del lenguaje mismo, englobado bajo el nombre de interpretación gramatical; segundo, la interpretación de las manifestaciones lingüísticas como expresiones de una subjetividad personal, reconocida como interpretación técnicopsicológica, y por último, trataremos de esbozar una síntesis de estas ideas con la configuración de una doctrina del arte de comprender.

\section{La interpretación gramatical}

En la interpretación gramatical, la tradición misma es importante en tanto pasado histórico que adviene lingüísticamente y que conecta a los hablantes entre sí. La tradición trae consigo un trasfondo que tiene un efecto beneficioso e inadvertido en el ejercicio de la interpretación. Recuperar esto es la tarea primordial de la hermenéutica, pues ninguna oración puede entenderse a menos que hayamos adoptado o reconstruido de antemano una herencia lingüística común. Se trata de un medio entre autor y lector que permite la comprensión. La interpretación gramatical debe prestar atención a la trama del texto, pues el significado de cada palabra debe verse a la luz del contexto en el que acontece. De lo contrario, carecería de sentido, ya que las palabras en sí mismas permanecen vagas y ambiguas, cuyas definiciones de diccionario dan solo ciertos usos que pueden ser delimitados, aclarados y complementados. Como señala Schleiermacher, la "tarea original" de los diccionarios es encontrar "la unidad perfecta de la palabra". ${ }^{24}$

La interpretación gramatical constituye la primera parte del método hermenéutico y tiene por finalidad encontrar el "sentido determinado" (bestimmter Sinn) de un cierto discurso "a partir del arte del lenguaje y con ayuda del lenguaje" (aus der Sprache und mit Hülfe der Sprache). ${ }^{25}$ Schleiermacher establece aquí un primer canon de la interpretación, el cual debe limitarse al lenguaje común entre escritor y lector. Es precisamente el objetivo de la interpretación gramatical lo que está definido o fijado con mayor precisión por esta vaga generalidad. Schleiermacher rechaza la idea de que la interpretación gramatical sea inferior a la interpretación técnico-psicológica. Por ejemplo, la interpretación gramatical puede descubrir muchas cosas de las que el propio autor es inconsciente y, por tanto, contribuir a una mayor comprensión. En este sentido, la comprensión nunca se logra de manera automática, sino que deber ser implementada en diferentes grados, a través del curso más o menos progresivo y circular de la interpretación. Según la tesis

24 Schleiermacher, Vorlesungen zur Hermeneutik und Kritik, 135.

25 Schleiermacher, Vorlesungen zur Hermeneutik und Kritik, 39. 
de Schleiermacher, el lenguaje es un universal a través del cual el hombre se singulariza, en que toda una historia vive en cada palabra hablada o escrita, y el eco de toda una tradición se escucha en cada texto. Por otra parte, Schleiermacher parte del supuesto de que no solo "cada escritor tiene su propio estilo", ${ }^{26}$ sino que cada individuo que habla tiene un estilo particular que se manifiesta en su lenguaje. El concepto de estilo no debe reducirse al concepto gramatical de forma o género literario, porque, incluso, dentro de un determinado género literario puede haber diferencias de estilo. ${ }^{27}$ En filosofía, por ejemplo, Nietzsche tiene un marcado estilo propio, que se nota en cada uno de sus escritos: su voluntad de ruptura, su fuerza polémica y su escritura fragmentaria buscan frente al discurso tradicional cambiar las formas de pensar, escribir y decir la filosofía misma. En literatura, por otra parte, con la lectura de las novelas de León Tolstói, podemos apreciar que su estilo literario es cercano al realismo, pues refleja con claridad las cosas bellas, veraces y fieles a la realidad, con una enseñanza totalmente sencilla que puede ser comprendida por cualquier público. Asimismo, la individualidad de un autor puede expresarse en una determinada selección de tópicos y temas que recorren sus obras.

\section{Interpretación técnico-psicológica}

Con la expresión "interpretación técnica", Schleiermacher se acerca a lo que los griegos llamaban téchne y que los latinos tradujeron con la expresión art. En opinión de Schleiermacher, la hermenéutica tiene la obligación de ir más allá de la letra del texto, para resaltar el espíritu de su autor a partir de los signos en los que se objetiva. Este aspecto técnico de la hermenéutica será llamado más adelante por Schleiermacher interpretación psicológica. La idea fundamental es clara: mientras que desde un punto de vista gramatical el autor está al servicio del lenguaje, desde un punto de vista psicológico el lenguaje está al servicio del autor.

Hay que hacer notar que hasta la lección que impartió en 1819 el interés de Schleiermacher se centraba en mayor medida en la interpretación gramatical, mientras que, a partir de 1820 hasta sus últimas lecciones de 1832-1833, la atención se centró en el aspecto técnico de la interpretación. En este giro, no debemos ver una degradación progresiva del método gramatical, sino una profundización del método técnico que, por otra parte, no se había abordado al comienzo de manera tan exhaustiva. En la recepción del pensamiento hermenéutico de Schleiermacher, el aspecto técnico-psicológico ciertamente ha tenido un significado más consistente y también ha llevado, en la línea de Gadamer, a una falsa consideración de este respecto del psicologismo.

Así como la interpretación gramatical no puede ignorar el horizonte universal de la totalidad del lenguaje, la interpretación técnica debe referirse firmemente a la totalidad de la personalidad del autor. Esto posibilita que, al producirse un tránsito de la interpretación gramatical a la técnica, la atención se vea desplazada hacia el individuo, entendido como fuente de origen de la obra. La tarea de la interpretación técnica es precisamente seguir los trazos de la obra hasta la personalidad del autor y la comprensión del vínculo que se extiende entre los dos extremos. Si bien puede haber un mínimo de interpretación gramatical, así como un mínimo de interpretación técnica, cada interpretación es una combinación de

26 Schleiermacher, Vorlesungen zur Hermeneutik und Kritik, 57.

27 Cf. Manfred Frank, “The Text and its Styles: Schleiermacher's Hermeneutic Theory of Language”, en The Subject and the Text: Essays on Literary Theory and Philosophy, ed. por Andrew Bowie (Cambridge: Cambridge University Press, 1997), 1-22. 
ambas. Aunque la interpretación gramatical y psicológica son aplicables en todas partes, a veces prevalece la primera y otras la segunda; por ejemplo, en las cartas personales, se requiere un mínimo de gramática versus un máximo de interpretación psicológica.

El método gira aquí en torno a los tres momentos principales de la redacción de un discurso en forma escrita: primero, está la idea, la decisión germinal del autor en la que se da el pensamiento fundamental de la obra; segundo, la meditación, la cual constituye el medio en que emerge la decisión; y tercero, la composición que emerge de las anteriores.$^{28}$ A partir de estas tres fases de la producción de un texto, la interpretación técnica se divide en dos momentos y complementarios: el psicológico y el estrictamente técnico. La interpretación psicológica tiene como objeto la decisión germinal, mientras que la técnica se ocupa de comprender el curso de los pensamientos que se desarrollan en forma lingüística. Está claro que ambos momentos de interpretación técnica nunca pueden desviarse de los resultados del método gramatical, ya que todo pensamiento o idea solo puede entenderse a través de su expresión en el lenguaje. Las preguntas que surgen ahora son ¿cómo surgió la idea fundamental del autor y se convirtió en una obra? y ¿cuál es la relación entre la obra y la vida de su autor? Estas interrogaciones ponen de relieve las tareas que son claves para el ejercicio de la interpretación técnica y psicológica. Entre ambas, aunque Schleiermacher utiliza estos términos como sinónimos, existe una distinción relativa tardía: la interpretación psicológica se refiere al carácter del autor y a sus virtudes más importantes, mientras que la técnica se ocupa principalmente de cómo se realiza un pensamiento o intención particular en la obra. Su relación es, por tanto, una relación general y única, por lo que la interpretación psicológica se centra en la totalidad de la vida del autor, el todo que ha vivido y expresado en su obra, en tanto que la técnica envuelve un determinado pensamiento o intención. Pero, según Schleiermacher, esta forma está determinada por la "decisión germinal" (Keimentschluß) del autor. ${ }^{29}$ Por ende, para comprender el texto en su conjunto, tenemos que captar esta intención original, lo que caracteriza la interpretación técnica como interpretación psicológica.

El papel más importante de la interpretación técnica es, por tanto, fijar la unidad interna de la obra, donde, así al menos lo parece, hay una cierta distinción entre el objeto real y el propósito de esta. El "objeto" real, en muchos casos, no es formulado ni expresado explícitamente por el autor, a diferencia del "objetivo" que sí es declarado. Como método más importante para captar el objeto, Schleiermacher sugiere prestar atención al principio y al final de la obra, y luego compararlos entre sí, lo cual debería hacer visible la unidad interna real de esta. Sin embargo, la culminación de la interpretación sigue siendo una reconstrucción de la experiencia mental del autor. En última instancia, el intérprete debe revivir la experiencia de autor de la que surgen la expresión, el estilo y la ejecución lingüística. En el curso de la interpretación, el término lingüístico debe remontarse a esta subjetividad del autor. Para Schleiermacher, el momento de la comprensión es, por tanto, una reconstrucción de la naturaleza experiencial del lenguaje.

\section{Kunstlehre}

Al inaugurar su lección de 1819, Schleiermacher advierte que una hermenéutica como "arte del comprender (Kunst des Verstehens) aún no existe", sino que solo es posible apreciar una dispersión del ejercicio de

28 Cf. Schleiermacher, Vorlesungen zur Hermeneutik und Kritik, 58-59.

29 Schleiermacher, Vorlesungen zur Hermeneutik und Kritik, 171. 
la interpretación a través de "muchas hermenéuticas especiales" (mehrere specielle Hermeneutiken). ${ }^{30}$ Un verdadero "arte de la interpretación" (Auslegungskunst) implica ponerse en posesión "de todas las condiciones de la comprensión" (aller Bedingungen des Verstehens). ${ }^{31}$ Su finalidad es la comprensión en el sentido más elevado, y solo se ha comprendido lo que se ha reconstruido en todas sus relaciones y contexto. Al extender la interpretación al ámbito psicológico, la hermenéutica de Schleiermacher se convierte en una "doctrina del arte" (Kunstlehre) de la interpretación. ${ }^{32}$ Se trata de un tránsito que va desde un ars interpretandi a la constitución de un método para la interpretación. Si bien su trabajo ha puesto al descubierto una serie de reglas muy precisas en el campo de la interpretación gramatical, estas ya no tienen el mismo alcance en la ejecución de la interpretación psicológica. En este nuevo escenario, como veremos más adelante, enfatiza la naturaleza adivinatoria del método hermenéutico, por el cual el procedimiento interpretativo deja de ser científico. La comprensión completa del habla o la escritura, así como la intuición por la que el intérprete capta la idea del autor, no puede tener reglas fáciles de enseñar y de aprender, sino que más bien se trata de un logro artístico que aplica tan solo pautas generales.

De esta manera, llegamos quizá a la contribución más valiosa de la teoría hermenéutica de Schleiermacher: por un lado, la interpretación gramatical puede formalizarse y legitimarse plenamente en pasos metodológicos externos, y por otro, la interpretación psicológica, que no se puede abordar de una manera científica, debe ser entendida como un arte (Kunst). La comprensión sería, entonces, el arte de combinar adecuadamente tanto el aspecto gramatical como el técnico-psicológico. Aquí emerge uno de los rasgos románticos del programa de Schleiermacher: comprender es "una tarea infinita" (eine unendliche Aufgabe), ${ }^{33}$ porque su objetivo es "captar" (begreifen) la individualidad del discurso que, sin embargo, solo puede ser abordado por aproximación progresiva. La interpretación se transforma en "reconstrucción" (Nachkonstruiren). ${ }^{34}$ La obra del intérprete es una imagen especular del autor de la obra, en el sentido de que su objetivo es la reconstrucción del texto. El trabajo de reconstrucción no es compilatorio, sino que consiste en el esfuerzo por seguir la progresión de la obra, para poder captar su génesis, el desarrollo y la lógica compositiva que la habita. En consecuencia, el proceso reconstructivo seguirá el movimiento descrito por el círculo hermenéutico, que describiremos más adelante, tanto en el nivel gramatical como en el técnico-psicológico.

\section{Dos métodos complementarios: comparativo y adivinatorio}

Si uno mira la práctica concreta del arte de la interpretación, Schleiermacher distingue dos procesos que operan fundamentalmente en el ejercicio cotidiano de comprensión, pero que son complementarios al modo de la interpretación gramatical y técnico-psicológica: se trata de los métodos comparativo y adivinatorio. El método comparativo supone "lo que ha de ser entendido como algo general", que luego encuentra lo peculiar "en la medida en que es comparado con lo otro". El método adivinatorio es "aquel

30 Schleiermacher, Vorlesungen zur Hermeneutik und Kritik, 119.

31 Schleiermacher, Vorlesungen zur Hermeneutik und Kritik, 73.

32 Schleiermacher, Vorlesungen zur Hermeneutik und Kritik, 116.

33 Schleiermacher, Vorlesungen zur Hermeneutik und Kritik, 6.

34 Schleiermacher, Vorlesungen zur Hermeneutik und Kritik, 128. 
en el cual uno mismo se transforma por igual en el otro, y busca aprender lo individual de manera inmediata". ${ }^{35}$ Por ejemplo, Schleiermacher expone que la adquisición del idioma primario por los niños se basa tanto en la adivinación como en la comparación de los usos de una palabra en varios contextos. ${ }^{36}$ Ambos métodos son complementarios, al igual que, en palabras de Schleiermacher, lo masculino y lo femenino van juntos en la antropología. La adivinación es definida como la "fuerza femenina" de la hermenéutica, que captura al individuo de forma inmediata por identidad, en tanto que el método comparativo constituye la "fuerza masculina", capaz de comprender la peculiaridad individual como parte de un universal, a través de la comparación con otros individuos. ${ }^{37} \mathrm{El}$ primero compara hechos objetivos entre sí, mientras el segundo intenta adivinar la combinación individual de un autor. El individuo solo puede ser reconocido como tal a través de una comparación con otros individuos, quienes, a su vez, deben ser captados por la identidad. Está claro que los dos métodos no pueden separarse, ya que la adivinación extrae su certeza y validez de la comparación, y los detalles con los que se compara lo universal del método comparativo son proporcionados por la adivinación. ${ }^{38}$

Es necesario que esta distinción entre ambos procedimientos no coincida con la distinción entre interpretación gramatical y psicológica. Incluso, desde el punto de vista gramatical, hay que adivinar ciertos hechos, y la comparación de dos autores entre sí también puede ser útil para determinar la peculiar individualidad de uno u otro. Esta complementariedad se registra en la fórmula plenamente positiva para la tarea hermenéutica, que se expresa bajo el siguiente procedimiento: "reconstruir el discurso pronunciado de una manera histórica y adivinatoria, objetiva y subjetiva". ${ }^{39}$ En este sentido, no hay duda de que la adivinación desempeña un papel mucho más importante en la interpretación psicológica que en la gramatical. Este es el lugar donde el intérprete se convierte en un ser virtuoso, es decir, un artista que tiene que empatizar con el secreto de toda interpretación: el secreto de la individualidad.

Si bien la interpretación gramatical puede seguir reglas claras que conducen a resultados definitivos, la interpretación psicológica, que considera el texto como una expresión de la vida y del espíritu de un autor, se basa más bien en el talento del intérprete. El significado psicológico ya no puede deducirse de un texto, sino que debe intuirse, sentirse y adivinarse. Solo a través de actos adivinatorios puede el intérprete entrar en el espíritu del autor. ${ }^{40}$ En la hermenéutica de Schleiermacher, la adivinación no tiene un significado místico, sino que hace referencia a lo que más tarde se llamará empatía. En el campo de la interpretación psicológica, la adivinación es ese estado de consciencia a través del cual el intérprete se transforma por igual en el otro, y busca aprender lo individual de manera inmediata" ${ }^{41}$ Aunque

35 Schleiermacher, Vorlesungen zur Hermeneutik und Kritik, 157.

36 Schleiermacher, Vorlesungen zur Hermeneutik und Kritik, 85.

37 Schleiermacher, Vorlesungen zur Hermeneutik und Kritik, 157.

38 Hugo Ochoa Disselkoen, "Comprender y explicar: Notas a partir de Schleiermacher", Universitas Philosophica 24, n. ${ }^{\circ}$ 49 (2007): 87.

39 Schleiermacher, Vorlesungen zur Hermeneutik und Kritik, 128.

40 Andreas Arndt reconstruye el método adivinatorio como una continuación de la concepción de la imaginación de Kant. La adivinación aparece como un momento esencial de un procedimiento totalizador, del cual ninguna interpretación de textos puede prescindir. Andreas Arndt, "Hermeneutik und Einbildungskraft", en Friedrich Schleiermachers Hermeneutik: Interpretationen und Perspektiven, ed. por Andreas Arndt y Jörg Dierken (Berlín, Walter de Gruyter, 2016), 119-128.

41 Schleiermacher, Vorlesungen zur Hermeneutik und Kritik, 157. 
en la práctica adivinatoria se pone de relieve, en consonancia con el pensamiento romántico, que la personalidad del autor casi nunca logra ser captada en toda su dimensión, un misterio fundamental e incomprensible se esconde detrás de cualquier creación.

Los métodos adivinatorio y comparativo no son independientes entre sí. Por una parte, en la interpretación, se deben comparar las observaciones realizadas a las obras escritas de un pueblo con todas las demás formas de objetivación de la cultura, en tanto son manifestaciones del mismo espíritu comunitario y, a su vez, del mismo espíritu individual. Por otra, en la interpretación, mediante la empatía, nos acercamos directamente a la otra persona y captamos su singularidad de una manera completamente personal y creativa, por tanto, de manera más artística. Entonces, el intérprete no actúa según las leyes científicas del entendimiento, sino solo sobre su talento personal y su genio específico. Cuanto mejor conoces a alguien, sostiene Schleiermacher, más analogías puedes encontrar. ${ }^{42}$ Aquí se revela uno de los aspectos singulares de la hermenéutica de Schleiermacher, que conlleva una doble exigencia: comprender al individuo desde el todo y al todo desde el individuo. ${ }^{43}$ Como veremos más adelante, se trata de un "círculo aparente". ${ }^{44} \mathrm{Si}$ bien la reconstrucción significa un progreso en la comprensión, la tarea de la comprensión es infinita.

\section{El círculo del comprender}

La noción del círculo (Zirkel) del comprender es una idea que ha tenido un gran éxito, especialmente en el contexto de la hermenéutica filosófica del siglo XX. La oposición entre totalidad y particularidad pertenece al conjunto conceptual de la individualidad. Es una estructura dialéctica fundamental que hace posible la comprensión y que aparece, en la hermenéutica de Schleiermacher, también bajo la forma de lo universal-particular. A partir del citado "borrador" de Halle de 1805, prevalece el término generalización (Allgemeinheit), puesto en oposición a particularidad (Eigenthümlichkeit). A penas la hermenéutica deja de ser un mero agregado de reglas mecánicas y se convierte en una doctrina del arte, debe afrontar la intuición de lo individual que solo puede entenderse con referencia a lo general, porque la "comprensión de lo individual está condicionado por la comprensión del todo", ${ }^{45}$ y viceversa, la comprensión de "lo individual es entendido solo técnicamente mediante la relación con la idea del todo". ${ }^{46}$ Sin embargo, a partir de la Hermenéutica general de 1809-1810, los términos que definen esta oposición son el todo (Ganze) y lo individual (Einzeln). Aquí Schleiermacher enuncia un principio de reciprocidad entre el todo de la herencia lingüística de un autor y su tiempo, y de las partes de las obras, las proposiciones y las palabras individuales.

Es plausible que el nuevo matiz léxico sea en parte atribuible a la lectura de dos textos que determinan una profundización de la reflexión de Schleiermacher sobre la hermenéutica, a saber, las obras de Friedrich Ast y Friedrich August Wolf, a quienes están dedicados los Discursos impartidos ante la Academia Prusiana de las Ciencias (1829), que puso en el centro de su reflexión el concepto de totalidad, el cual será entendido como

42 Schleiermacher, Vorlesungen zur Hermeneutik und Kritik, 169-170.

43 Schleiermacher, Vorlesungen zur Hermeneutik und Kritik, 131.

44 Schleiermacher, Vorlesungen zur Hermeneutik und Kritik, 129.

45 Schleiermacher, Vorlesungen zur Hermeneutik und Kritik, 29.

46 Schleiermacher, Vorlesungen zur Hermeneutik und Kritik, 104. 
unidad espiritual superior, revelado en el lenguaje de manera privilegiada. Se puede suponer que Friedrich Ast en particular llamó la atención de Schleiermacher sobre la importancia de este fenómeno. Ciertamente no es casualidad que la representación de este círculo ocupe casi todo el segundo Discurso. Para Ast, afirma Schleiermacher, "el pensamiento de que todo lo individual puede ser únicamente comprendido mediante el todo, y, por tanto, toda explicación de lo individual presupone ya la comprensión del todo". ${ }^{47}$ Si bien Ast vio el fenómeno de la circularidad, no logró integrarlo orgánicamente en el concepto de comprensión.

Solo cuando uno se da cuenta de que un gran número de reglas hermenéuticas se basan más o menos en este principio hermenéutico, el círculo se vuelve un concepto hermenéuticamente productivo; en el fondo, el intérprete primero debe reconstruir gradualmente una idea del todo. En la interpretación psicológica, la relación entre el todo y las partes es fundamentalmente dialéctica: la obra individual se debe a un acto del autor y vuelve a "un momento germinal fecundo de su autor" ${ }^{8}$ Los pensamientos individuales, es decir, los momentos emergentes de la vida del autor, solo pueden entenderse en el contexto de todo su mundo de pensamiento.

Ningún discurso puede entenderse por sí solo, sino en un contexto más amplio. Su comprensión requiere un conocimiento previo del autor y de su objeto. Por tanto, "no solo la comprensión del todo está condicionada por la comprensión de lo individual, sino también esta por la del todo (umgekehrt das des Einzelnen durch das des Ganzen)" " ${ }^{49}$ Schleiermacher termina la introducción de su Hermenéutica general de 1819 con la siguiente frase: "La comprensión surge en la medida en que ambas operaciones se complementan, en que la imagen de la totalidad (das Bild des Ganzen) lo hace con la comprensión de lo individual (des Einzelnen) y en que lo individual se comprenda completamente tanto más cuanto que tengamos una visión general del conjunto". ${ }^{50}$

En síntesis, el círculo hermenéutico tiene un valor fundamental si se descifra a la luz de los contenidos filosóficos de la teoría de Schleiermacher y, en particular, de la individualidad. El individuo, de hecho, está en el centro del interés hermenéutico, pero esto dificulta encontrar una verdad última e invariable. La circularidad expresa el carácter infinito y siempre aproximado de la interpretación y podría considerarse inherente al problema de la comprensión en sí, ya que se extiende tanto al objeto a comprender como a los medios de que dispone el intérprete. Sin embargo, es importante señalar que no se trata de un círculo vicioso, sino de una posibilidad de una perfección cada vez mayor en la comprensión del individuo.

\section{Comprender mejor}

La primera referencia a este enunciado, probablemente el más célebre de Schleiermacher, la encontramos, en el conocido pasaje al comienzo de la "Dialéctica trascendental" de la Crítica de la razón pura. Allí Kant asume que un intérprete puede "comprender mejor" analizando cuidadosamente los términos utilizados

47 Schleiermacher, Los discursos sobre hermenéutica, 89.

48 Schleiermacher, Los discursos sobre hermenéutica, 99.

49 Schleiermacher, Vorlesungen zur Hermeneutik und Kritik, 78.

50 Schleiermacher, Vorlesungen zur Hermeneutik und Kritik, 78. 
por un autor. ${ }^{51}$ En segundo lugar, encontramos esta expresión en la última de las "Lecciones sobre el destino del sabio", de Fichte, quien tuvo una gran influencia en los primeros románticos. Fichte discute la doctrina de Rousseau, mostrando, primero, las contradicciones de su pensamiento y, luego, buscando una solución "mejor de lo que él mismo se entendió". ${ }^{22}$ En tercer lugar, esta expresión la encontramos en la filosofía de la identidad de Schelling basada en la teoría de la creación inconsciente. Así como la naturaleza crea de forma inconsciente, las personas producen obras de arte con cierto grado de olvido de sí mismas. Schelling asume que los seres humanos y la naturaleza comparten la misma fuerza creativa elemental. ${ }^{53}$

Si uno clasificara la teoría romántica temprana de la comprensión de acuerdo con estos tres modelos, en palabras de Ernst Behler, tanto Friedrich Schlegel como Novalis se acercarían a la posición representada por Fichte; en cambio, Schleiermacher se aproxima a la teoría de la creación inconsciente de Schelling. ${ }^{54}$ En sus Discursos, señala que es posible comprender un discurso "mejor que su autor", en tanto que la tarea de la hermenéutica busca "comprender a un autor mejor de lo que él mismo podría dar cuenta de sí mismo". ${ }^{55}$ La totalidad de estos puntos de vista, de los que un autor difícilmente será plenamente consciente al escribir su discurso u obra, hace comprensible por qué Schleiermacher afirma como el objetivo de toda interpretación comprender a un autor "de manera más elevada" y "más informada." ${ }^{6}$

Desde los primeros aforismos hasta las últimas lecciones, esta idea recorre, con algunas variaciones, las venas del pensamiento hermenéutico de Schleiermacher. ${ }^{57}$ Alcanzar adecuadamente el sentido de la máxima "comprender a un autor mejor de lo que él mismo se entendió" requiere identificar las partes íntimas de la obra de un autor y reconocer la relación con su contexto. Como lo afirma tempranamente, se trata de buscar "el secreto más profundo de la individualidad y reconstruir (nachkonstruiren) el individuo de manera artística (künstliche Weise)" ${ }^{58}$ Schleiermacher también cuenta con la posibilidad de que el autor lleve a cabo este proceso de autoconsciencia de sí mismo para que se convierta reflexivamente en su propio lector. Por supuesto, es mucho más difícil aplicar la máxima en el lado psicológico, porque aquí no es el lenguaje objetivo el que debe ser consciente, sino los procesos creativos inconscientes. Esto, naturalmente, suscita la cuestión de la posible dependencia del pensamiento de Schleiermacher a la estética del genio.

\section{A modo de conclusión: para una hermenéutica de las ciencias humanas}

Si bien se puede argumentar que existe una estrecha conexión entre la hermenéutica y las ciencias humanas, la hermenéutica tiene una historia mucho más larga, como hemos visto, que comenzó como

51 Immanuel Kant, Kritik der reinen Vernunft (Hamburgo: Felix Meiner, 2010), 246.

52 Johann Fichte, Zur theoretischen Philosophie Fichte (Berlín: Walter de Gruyter, 1971), 265.

53 Friedrich Schelling, Historisch-kritische Gesamtausgabe (Stuttgart: Frommann-Holzboog, 1976), 169.

54 Ernst Behler, Frühromantik (Berlín: Walter de Gruyter, 1992), 272.

55 Schleiermacher, Los discursos sobre hermenéutica, 81.

56 Schleiermacher, Vorlesungen zur Hermeneutik und Kritik, 34.

57 Schleiermacher, Vorlesungen zur Hermeneutik und Kritik, 34, 75, 114, 128, 133.

58 Friedrich Schleiermacher, “Garve's letzt noch von ihm selbst herausgegebene Schriften”, en Schriften aus der Berliner Zeit. 1800-1802, KGA I/3 (Berlín: Walter de Gruyter, 1988), 69. 
una teoría de la interpretación de los textos sagrados y que, gracias a Schleiermacher, extendió su competencia, gradualmente, al trabajo de la interpretación de una gama mucho más amplia de textos. De hecho, cuando uno mira lo que sucede actualmente con la hermenéutica, su principal preocupación son las humanidades: la historia, el derecho, la literatura y las artes. Por tanto, la hermenéutica se ofrece como una importante piedra de toque intelectual para la investigación de las ciencias humanas contemporáneas.

La hermenéutica, desde el siglo XIX en adelante, ha estado vinculada al desarrollo de las humanidades, en vista de resolver el problema de la distancia histórica del investigador y el nuevo contexto de apropiación. Fue Wilhelm Dilthey (1833-1911) quien sostuvo que las herramientas intelectuales de la hermenéutica de Schleiermacher se adaptan a la interpretación de la acción humana y de la historia. ${ }^{59}$ En la actualidad, creo que hay un margen considerable para desarrollar y ampliar las posibilidades de la teoría hermenéutica para su aplicación a las actividades de los investigadores en las ciencias humanas. En realidad, parte del potencial de este desarrollo ya se encuentra en los escritos de algunos de los teóricos clave. ${ }^{60}$ Por tanto, es sumamente interesante volver a examinar algunos de los conceptos primarios de la hermenéutica y observar de qué manera puede contribuir al desarrollo de la investigación cualitativa en psicología, educación, historia, estética, entre otras.

Examinaremos la noción de círculo hermenéutico, que hemos explorado en su versión preliminar en la obra de Schleiermacher. Se trata de la idea que ha tenido una mayor repercusión en las ciencias sociales, en tanto que defiende la relación dinámica entre la parte y el todo. Si bien esto ha sido criticado desde una perspectiva lógica debido a su circularidad inherente, analíticamente respecto de describir el proceso de interpretación parece potente. Claramente, parte del atractivo de esta expresión radica en que habla de un estilo de análisis no lineal y de la posibilidad de profundizar constantemente en la interpretación. Por supuesto, hay que equilibrar esto con una gran dosis de pragmatismo; es posible que nunca se llegue a la interpretación final, ya que teóricamente el círculo podría continuar para siempre.

El círculo hermenéutico describe la relación entre el intérprete y los diferentes aspectos de su "objeto". El intérprete empieza en un punto determinado del círculo, imbuido en sus preocupaciones, influenciado por sus ideas preconcebidas, moldeado por su experiencia y pericia. Al moverse de esta posición, el intérprete debe reconocer sus ideas preconcebidas antes de ir al encuentro de una obra o de un participante de la investigación que está al otro lado del círculo. Cualesquiera que sean las preocupaciones o posiciones anteriores del intérprete, este pasa de un punto en el que estaba en el centro de atención a uno en el que la obra o el participante se transforma en uno nuevo. Se debe prestar atención a su historia para que emerjan las experiencias, lo cual requiere, en el caso de las investigaciones sociales, una intensa atención y compromiso con el participante mientras da a conocer sus experiencias. Por supuesto, aquí se ofrece una versión simplificada de lo que es un proceso dinámico y complejo al nivel del trabajo de campo.

Si seguimos de cerca la trayectoria del investigador social, veremos que la historia no termina allí, sino que se desplaza también al trabajo de escritura que da cuenta de los resultados de la investigación. Habiendo

59 Cf. Wilhelm Dilthey, Psicología y teoría del conocimiento (México: Fondo de Cultura Económica, 2014), 199.

60 Hans-Georg Gadamer, El problema de la conciencia histórica (Madrid: Tecnos, 2000); Jürgen Habermas, La lógica de las ciencias sociales (Madrid: Tecnos, 2000); Zygmunt Bauman, La hermenéutica y las ciencias sociales (Buenos Aires: Nueva Visión, 2007); Clifford Geertz, La interpretación de las culturas (Barcelona: Gedisa, 2003); Babette Babich, Hermeneutic Philosophies of Social Science (Berlín: Walter de Gruyter, 2017). 
concluido el trabajo de campo, el investigador continúa su viaje alrededor del círculo de regreso al lugar donde comenzó. Al regresar a casa y al analizar el material que fue recopilado, puede reconocer que su posición ha cambiado, influenciado por el encuentro con el grupo humano y su narración. Si el investigador retoma la conversación con el participante, mientras pasa revista a su historia, la conversación toma una nueva dimensión y probablemente un sentido más amplio.

Tanto la idea cartesiana como la idea kantiana de la ciencia, que sentaron las bases del desarrollo científico moderno, establecieron que las reflexiones sobre el método y los límites de la razón tenían que preceder a todas las investigaciones. En la nueva forma de entender la "ciencia", su tarea primera no es descubrir la verdad, sino establecer un sistema de reglas para evitar el error y determinar los límites del método. En esencia, no es una búsqueda de una serie de primeros principios, sino que está guiada por el espíritu pragmático de las reflexiones metodológicas sobre las necesidades y los intereses predeterminados de la investigación positiva. Ante este espíritu, la hermenéutica de Schleiermacher ofrece una alternativa.

La hermenéutica de Schleiermacher es, sin duda, uno de los puntos de referencia más importantes en el debate hermenéutico contemporáneo, y así se convierte en un instrumento de crítica gracias a su capacidad de "reconstruir" (nachkonstruiren) el discurso. Surge el esquema de una crítica genética, gracias a la cual el intérprete encuentra y hace consciente lo que no se reflejó en la composición de un texto. El principio de "comprender mejor" (besser zu verstehen) propone abordar aquellos aspectos aún pendientes de descifrar de la obra de un autor. Asimismo, como lo ha mostrado la crítica literaria del siglo XX, y en particular la teoría de la recepción, la obra es infinitamente abierta y, por tanto, no reducible a la intención del autor. Confío en que este artículo arroje un poco de luz sobre su concepción tanto desde una perspectiva problemática como sistemática. De esta manera, el lector puede obtener una imagen multifacética de su hermenéutica frente a su trasfondo histórico y a los problemas actuales de las teorías de interpretación.

\section{Bibliografía}

Arndt, Andreas y Jörg Dierken. Friedrich Schleiermachers Hermeneutik: Interpretationen und Perspektiven. Berlín: Walter de Gruyter, 2016.

Arndt, Andreas. "Hermeneutik und Einbildungskraft". En Friedrich Schleiermachers Hermeneutik, editado por Andreas Arndt y Jörg Dierken, 119-128. Berlín: Walter de Gruyter, 2016.

Arndt, Andreas. Schleiermacher als Philosoph. Berlín: Walter de Gruyter, 2013.

Babich, Babette. Hermeneutic Philosophies of Social Science. Berlín: Walter de Gruyter, 2017.

Bauman, Zygmunt. La hermenéutica y las ciencias sociales. Buenos Aires: Nueva Visión, 2007.

Behler, Ernst. Frühromantik. Berlín: Walter de Gruyter, 1992.

Dilthey, Wilhelm. Psicología y teoría del conocimiento. México: Fondo de Cultura Económica, 2014.

Fichte, Johann. Zur theoretischen Philosophie Fichte. Berlín: Walter de Gruyter, 1971.

Flamarique, Lourdes. Introducción a Los discursos sobre hermenéutica, de Friedrich Schleiermacher, 5-45. Pamplona: Universidad de Navarra, 1999.

Frank, Manfred. “The Text and its Styles: Schleiermacher's Hermeneutic Theory of Language”. En The Subject and the Text: Essays on Literary Theory and Philosophy, editado por Andrew Bowie, 1-22. Cambridge: Cambridge University Press, 1997.

Gadamer, Hans-Georg. El problema de la consciencia histórica. Madrid: Tecnos, 2000.

Gadamer, Hans-Georg. Hermeneutik I: Wahrheit und Methode. Grundzüge einer philosophischen Hermeneutik. Tubinga: Mohr-Siebeck, 2010. 
Geertz, Clifford. La interpretación de las culturas. Barcelona: Gedisa, 2003.

Grondin, Jean. Introducción a la hermenéutica filosófica. Barcelona: Herder, 2002.

Habermas, Jürgen. La lógica de las ciencias sociales. Madrid: Tecnos, 2000.

Jaeschke, Walter y Andreas Arndt. Die Klassische Deutsche Philosophie nach Kant: Systeme der reinen Vernunft und ihre Kritik 1785-1845. Múnich: Beck, 2012.

Kant, Immanuel. Kritik der reinen Vernunft. Hamburgo: Felix Meiner, 2010.

Nowak, Kurt. Schleiermacher und die Frühromantik: Eine literaturgeschichtliche Studie zum romantischen Religionsverständnis und Menschenbild am Ende des 18. Jahrhunderts in Deutschland. Gotinga: Vandenhoeck \& Ruprecht, 1986.

Nowak, Kurt. Schleiermacher: Leben, Werk und Wirkung. Gotinga: Vandenhoeck \& Ruprecht, 2001.

Ochoa Disselkoen, Hugo. "Comprender y explicar: Notas a partir de Schleiermacher". Universitas Philosophica 24, n. ${ }^{\circ} 49$ (2007): 79-94.

Schelling, Friedrich. Historisch-kritische Gesamtausgabe. Stuttgart: Frommann-Holzboog, 1976.

Schleiermacher, Friedrich. Briefwechsel 1796-1798, KGA V/2. Berlín: Walter de Gruyter, 1988.

Schleiermacher, Friedrich. Briefwechsel 1804-1806, KGA V/8. Berlín: Walter de Gruyter, 1988.

Schleiermacher, Friedrich. "Garve's letzt noch von ihm selbst herausgegebene Schriften". En Schriften aus der Berliner Zeit. 1800-1802, KGA I/3, 65-72. Berlín: Walter de Gruyter, 1988.

Schleiermacher, Friedrich. Hermeneutik. Heidelberg: Abhandlungen der Heidelberger Akademie der Wissenschaften, 1959.

Schleiermacher, Friedrich. Hermeneutik und Kritik. Fráncfort del Meno: Suhrkamp, 1977.

Schleiermacher, Friedrich. Hermeneutik und Kritik mit besonderer Beziehung auf das Neue Testament. Berlín: Reimer, 1838.

Schleiermacher, Friedrich. Los discursos sobre hermenéutica. Pamplona: Universidad de Navarra, 1999.

Schleiermacher, Friedrich. Vorlesungen zur Hermeneutik und Kritik, KGA II/6. Berlín: Walter de Gruyter, 2012.

Thouard, Denis. "Die Sprachphilosophie der Hermeneutik". En Friedrich Schleiermachers, Hermeneutik, Interpretationen und Perspektiven, editado por Andreas Arndt y Jörg Dierken, 85-100. Berlín: Walter de Gruyter, 2016.

Virmond, Wolfgang. Introducción a Vorlesungen zur Hermeneutik und Kritik, KGA II/6, de Friedrich Schleiermacher, VII-LII. Berlín: Walter de Gruyter, 2012. 